\title{
Analytic Formulas to Calculate Symmetric Brothers of a Node in a Perfect Binary Tree
}

\author{
Xingbo WANG ${ }^{1,2,3} \&$ Zhen SHEN $^{1}$ \\ ${ }^{1}$ Department of Mechatronic Engineering, Foshan University, Foshan City, PRC 528000 \\ ${ }^{2}$ Guangdong Engineering Center of Information Security for Intelligent Manufacturing System, PRC \\ ${ }^{3}$ State Key Laboratory of Mathematical Engineering and Advanced Computing,Wuxi City, PRC
}

Correspondence: Xingbo WANG, Department of Mechatronic Engineering, Foshan University, Foshan City, PRC, 528000

Received: July 16, 2018 Accepted: August 1, 2018 Online Published: August 8, 2018

doi:10.5539/jmr.v10n5p45 URL: https://doi.org/10.5539/jmr.v10n5p45

The research is financed by the State Key Laboratory of Mathematical Engineering and Advanced Computing under Open Project Program No.2017A01, Department of Guangdong Science and Technology under project 2015A010104011, Foshan Bureau of Science and Technology under projects 2016 AG100311

\begin{abstract}
The paper puts forward and solves the problem of calculating the symmetric brother nodes of a given node in a perfect binary tree. By analyzing the relationships between a node and its ancestors, the position at which a node lies in term of the subtree rooted by an ancestor is expressed with a mathematical formula and consequently the mathematical formula to express its symmetric nodes is derived out. The formulas enable an easy calculation of a node and its symmetric nodes in the perfect binary tree.
\end{abstract}

Keywords: perfect binary tree, symmetric node, calculation

\section{Introduction}

In a perfect binary tree, whose definition is seen at page 877 in Paul's dictionary (Paul E Black,2004) and as illustrated in figure 1, an internal node might have a father, a grandfather or even ancestors of higher generations, as described by certain entries in the handbooks edited by Rosen K (Rosen K, 2000) and by Dinesh P M \& Sartaj Sahni (Dinesh 2004). On a level of a perfect binary tree, it is known that, a node must have a brother that shares a father with it; it also has cousins that share grandfather or ancestors of higher generations. Literatures of studying on the ancestors or descendants of a node in a tree can be frequently found in library. For example, Berkman's paper (Berkman O \& Vishkin U, 1994) and Papamichail's paper (Papamichail D \& Caputi T \& Papamichail G, 2014) are on how to find an ancestor of a node while the paper (Knight S, Kim H, Meghanathan N, et al. 2007) and the paper (Fly P, Meghanathan N, Isokpehi R, 2011) are on analysis of ancestor-descendant relationships. However it is a pity that one can hardly find a bibliography on how to find a brother or a cousin of a node in a tree. A recent study comes across a problem of finding the symmetric brothers (or cousins) for a given node. For example, given the node $(3,2)$ in figure 1 , it has, as its symmetric brother nodes, the node $(3,1)$ that shares a grandfather $(1,0)$, the node $(3,3)$ that shares a father $(2,1)$ and the node $(3,5)$ that shares the root $(0,0)$. Obviously, when the binary tree is big enough, it is really difficult for one to solve the problem in a simple way. Since there is no referable literature, this article solves the problem.

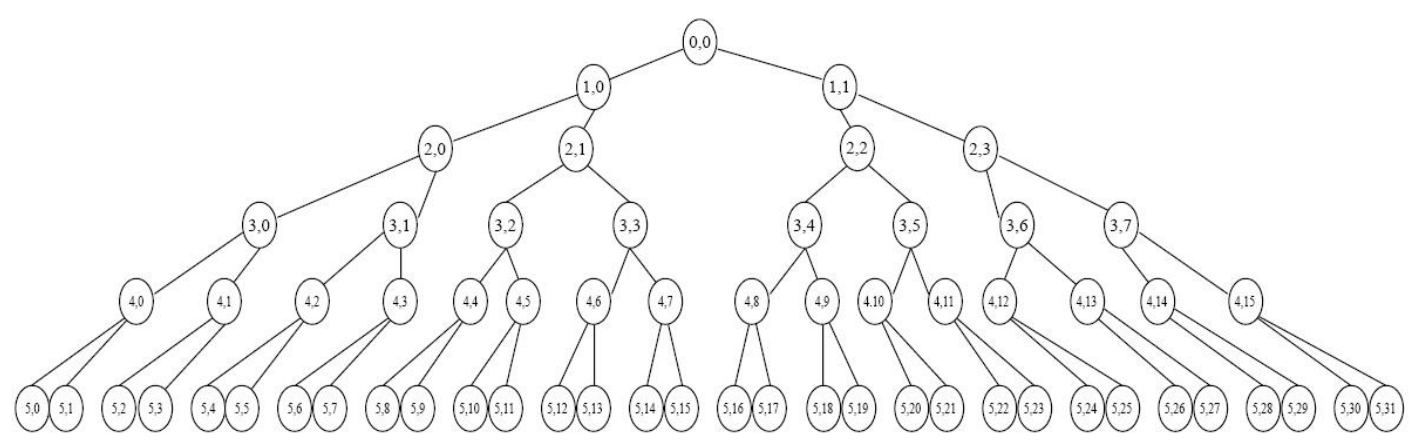

Figure 1. A perfect binary tree from level 0 to level 5 


\section{Preliminaries}

This section lists the preliminaries that include definitions, symbols and lemmas, which are necessary for later sections.

\subsection{Symbols and Notations}

A perfect binary tree $\boldsymbol{T}$, which is defined at page 877 in Paul's dictionary, is a binary tree with all leaf nodes at the same depth. All internal nodes have degree 2. Definitions related with the father, the ancestors and the descendants of a node in a binary trees can be seen by certain entries in the Rosen's and Dinesh's handbooks. In this paper, a node of a binary tree refers to either a vertex or a leaf, and a brother means a brother node or a cousin node. For convenience, symbol $N_{(k, j)}$ is the node at the position $j$ on level $k$ of $\boldsymbol{T}$, where $k>0$ and $0 \leq j \leq 2^{k}-1$. Number of the level by default begins with zero and index of the position also by default begins with zero; symbol $T_{N_{(k, j)}}$ denotes a subtree whose root is $N_{(k, j)}$ and symbol $N_{(i, \omega)}^{N_{(k, j)}}$ denotes the node at the position $\omega$ on level $i$ of $T_{N_{(k, j)} .}$. Node $N_{(i, \omega)}^{N_{(k, j)}}$ and node $N_{\left(i, 2^{i}-1-\omega\right)}^{N_{(k, j)}}$ are geometrically symmetric on level $i$ with $i>0$ thus they are called symmetric nodes. Accordingly, the path that connects $N_{(k, j)}$ and $N_{(i, \omega)}^{N_{(k, j)}}$ is said to be symmetric to the path that connects $N_{(k, j)}$ and $N_{\left(i, 2^{i}-1-\omega\right)}^{N_{(k, j)}}$. The path from $N_{(i, \omega)}^{N_{(k, j)}}$ to $N_{(k, j)}$ and to $N_{\left(i, 2^{i}-1-\omega\right)}^{N_{(k, j)}}$ is called a symmetric path. Symbol $\lfloor x\rfloor$ is the floor function, an integer function of real number $x$ that satisfies inequality $x-1<\lfloor x\rfloor \leq x$, or equivalently $\lfloor x\rfloor \leq x<\lfloor x\rfloor+1$. Symbol mod is the modulo operation and expression $r=a \bmod b$ means $a \equiv r(\bmod b)$.

\subsection{Lemmas}

Lemma 1 (See in (WANG X B, 2015)) Let $N_{(m, \alpha)}$ be the node at position $\alpha$ on level $m$ of a perfect binary tree $\boldsymbol{T}$ with $m>0$ and $0 \leq \alpha \leq 2^{m}-1$; then the direct ancestors of $N_{(m, \alpha)}$ are calculated by $N_{(m-i, \sigma(i, \alpha))}$, where $\sigma(i, \alpha)=\left\lfloor\frac{\alpha}{2^{i}}\right\rfloor(i>0)$. The level $i$ with $i \geq 0$ of $T_{N_{(m, \alpha)}}$ is the level $m+i$ of $\boldsymbol{T}$ and $N_{(i, \omega)}^{N_{(m, \alpha)}}=N_{\left(m+i, 2^{i} \alpha+\omega\right)}$.

\section{Main Results and Proofs}

Theorem 1 Let $N_{(k, j)}$ be a node of a perfect binary tree $\boldsymbol{T}$ with $k>0$; then there are $k$ symmetric paths connecting $N_{(k, j)}$ and its symmetric nodes. In another word, $N_{(k, j)}$ has $k$ symmetric brothers on level $k$ in $\boldsymbol{T}$.

Proof $N_{(k, j)}$ lies on level $k$; hence it has totally $k$ ancestors in the tree. Since each of its ancestors can produce a subtree in which $N_{(k, j)}$ has a symmetric brother node, it is sure that it has $k$ symmetric brothers on level $k$ and there are $k$ paths connecting $N_{(k, j)}$ to its symmetric nodes.

Example 1. Take in figure 1 node $N_{(1,0)}$. It has a symmetric node $N_{(1,1)}$; the symmetric path is $\left\{N_{(1,0)}, N_{(0,0)}, N_{(1,1)}\right\}$. Take another node $N_{(2,0)}$. It has a symmetric node $N_{(2,3)}$ in term of $\boldsymbol{T}$, and a symmetric node $N_{(2,1)}$ in term of $T_{N_{(1,0)}}$. Consequently there are 2 symmetric paths connecting $N_{(2,0)}$ to its symmetric nodes $N_{(2,3)}$ and $N_{(2,1)}$. The two symmetric paths are $\left\{N_{(2,0)}, N_{(1,0)}, N_{(0,0)}, N_{(1,1)}, N_{(2,3)}\right\}$ and $\left\{N_{(2,0)}, N_{(1,0)}, N_{(2,1)}\right\}$.

Theorem 2 Let $N_{(k, j)}$ be a node of a perfect binary tree $\boldsymbol{T}$ with $k>0$. If $N_{(k, j)}$ is a left node, then all its symmetric brothers must be right ones; or vice versa, if $N_{(k, j)}$ is a right node, then all its symmetric brothers must be left ones.

Proof By Theorem 1, in $\boldsymbol{T}$ there are $k$ nodes each of which is the root of a subtree in which $N_{(k, j)}$ lies. Suppose $N_{(k, j)}=N_{(i, \omega)}^{A}$ lies in $T_{A}$ with $i>0$; then by definition its symmetric brother is the node $N_{\left(i, 2^{i}-1-\omega\right)}^{A}$. Note that, by binary representation it yields

$$
\omega+\left(2^{i}-1-\omega\right)=2^{i}-1=(\underbrace{11 \ldots 1}_{i-1})_{2}
$$

it knows that, the most significant bit (msb) of $\omega$ must be different from the msb of $2^{i}-1-\omega$, which means a left node $N_{(i, \omega)}^{A}$ leads to a right symmetric node $N_{\left(i, 2^{i}-1-\omega\right)}^{A}$, or vice versa, a right node $N_{(i, \omega)}^{A}$ leads to a left symmetric node $N_{\left(i, 2^{i}-1-\omega\right)}^{A}$.

Theorem 3 Let $N_{(k, j)}$ be a node in a perfect binary tree $T$ with $k>0$ and $A_{1}, A_{2}, \cdots, A_{k}$ be the father, the grandfather and the so-forth ancestors of $N_{(k, j)}$ respectively; then $N_{(k, j)}$ is the node $N_{\left(1, j \bmod 2^{1}\right)}^{A_{1}}$ in $T_{A_{1}}$, the node $N_{\left(2, j \bmod 2^{2}\right)}^{A_{2}}$ in $T_{A_{2}}$, and so forth, the node $N_{\left(i, j \bmod 2^{i}\right)}^{A_{i}}$ in $T_{A_{i}}$ until $i=k$. Consequently, its symmetric brothers in $T_{A_{1}}, T_{A_{2}}, \cdots, T_{A_{i}}, \cdots, T_{A_{k}}$ are respectively the nodes $N_{\left(1,2^{1}-1-j \bmod 2^{1}\right)}^{A_{1}}, N_{\left(2,2^{2}-1-j \bmod 2^{2}\right)}^{A_{2}}, \cdots, N_{\left(i, 2^{i}-1-j \bmod 2^{i}\right)}^{A_{i}}, \cdots$, and $N_{\left(k, 2^{k}-1-j \bmod 2^{k}\right)}^{A_{k}}$, or equivalently,

$$
N_{\left(1,2\left\lfloor\frac{j}{2}\right\rfloor+1-j\right)}^{A_{1}}, N_{\left(2,2^{2}\left\lfloor\frac{j}{2^{2}}\right\rfloor+2^{2}-1-j\right)}^{A_{2}}, \ldots, N_{\left(i, 2^{i}\left\lfloor\frac{j}{2^{i}}\right\rfloor+2^{i}-1-j\right)}^{A_{i}}, \ldots, N_{\left(k, 2^{k}\left\lfloor\frac{j}{2^{k}}\right\rfloor+2^{k}-1-j\right)}^{A_{k}}
$$


Proof By Lemma 1, it knows that, if $j \bmod 2=0$ then $N_{(k, j)}=N_{(1,0)}^{A_{1}}$ else $N_{(k, j)}=N_{(1,1)}^{A_{1}}$, or equivalently

$$
N_{(k, j)}=N_{(1, j \bmod 2)}^{A_{1}}
$$

Likewise, it holds

$$
N_{(k, j)}=N_{\left(2, j \bmod 2^{2}\right)}^{A_{2}}=N_{\left(3, j \bmod 2^{3}\right)}^{A_{3}}=\cdots=N_{\left(k, j \bmod 2^{k}\right)}^{A_{k}}
$$

Hence its corresponding symmetric brothers in $T_{A_{1}}, T_{A_{2}}, \cdots, T_{A_{i}}, \cdots, T_{A_{k}}$ are respectively given by

$$
N_{\left(1,2^{1}-1-j \bmod 2^{1}\right)}^{A_{1}}, N_{\left(2,2^{2}-1-j \bmod 2^{2}\right)}^{A_{2}}, \cdots, N_{\left(i, 2^{i}-1-j \bmod 2^{i}\right)}^{A_{i}}, \cdots, N_{\left(k, 2^{k}-1-j \bmod 2^{k}\right)}^{A_{k}}
$$

By formula $a \bmod b=a-b\left\lfloor\frac{a}{b}\right\rfloor$, it holds

$$
\begin{aligned}
& N_{\left(1,2^{1}-1-j \bmod 2^{1}\right)}^{A_{1}}=N_{\left(1,2\left\lfloor\frac{j}{2^{\prime}}\right\rfloor+2^{1}-1-j\right)}^{A_{1}}, N_{\left(2,2^{2}-1-j \bmod 2^{2}\right)}^{A_{2}}=N_{\left(2,2^{2}\left\lfloor\frac{j}{2^{2}}\right\rfloor+2^{2}-1-j\right)}^{A_{2}}, \cdots \\
& N_{\left(i, 2^{i}-1-j \bmod 2^{i}\right)}^{A_{i}}=N_{\left(i, 2^{i}\left\lfloor\frac{j}{2^{i}}\right\rfloor+2^{i}-1-j\right)}^{A_{A^{2}}}, \cdots, N_{\left(k, 2^{k}-1-j \bmod 2^{k}\right)}^{A_{k}}=N_{\left(k, 2^{k}\left\lfloor\frac{j}{2^{k}}\right\rfloor+2^{k}-1-j\right)}^{A^{k}},
\end{aligned}
$$

Corollary 1 Let $N_{(k, j)}$ be a node in a perfect binary tree $T$ with $k>0$ and $A_{1}, A_{2}, \cdots, A_{k}$ be the father, the grandfather and the so-forth ancestors of $N_{(k, j)}$ respectively; then the symmetric brothers of $N_{(k, j)}$ in the subtrees rooted by $A_{1}, A_{2}, \ldots, A_{k}$ respectively are calculated in term of nodes on level $k$ of $T$ by

$$
N_{\left(k, 2\left\lfloor\frac{j}{2}\right\rfloor+1-j \bmod 2\right)}, N_{\left(k, 2^{2}\left\lfloor\frac{j}{2^{2}}\right\rfloor+2^{2}-1-j \bmod 2^{2}\right)}, \cdots, N_{\left(k, 2^{i}\left\lfloor\frac{j}{2^{i}}\right\rfloor+2^{i}-1-j \bmod 2^{i}\right)}, \cdots, N_{\left(k, 2^{k}\left\lfloor\frac{j}{2^{k}}\right\rfloor+2^{k}-1-j \bmod 2^{k}\right)}
$$

or equivalently

$$
N_{\left.\left(k, 2^{1+1}\left\lfloor\frac{j}{2}\right\rfloor+1-j\right)\right)}, N_{\left(k, 2^{2+1}\left\lfloor\frac{j}{2^{2}}\right\rfloor+2^{2}-1-j\right)}, \ldots, N_{\left(k, 2^{i+1}\left\lfloor\frac{j}{2^{i}}\right\rfloor+2^{i}-1-j\right)}, \ldots, N_{\left(k, 2^{k+1}\left\lfloor\frac{j}{2^{k}}\right\rfloor+2^{k}-1-j\right)}
$$

Proof By Lemma 1, it holds in $T_{N_{(k, j)}}$

$$
N_{(i, \omega)}^{N_{(k, j)}}=N_{\left(k+i, 2^{i} j+\omega\right)}
$$

and

$$
A_{1}=N_{\left(k-1,\left\lfloor\frac{j}{2}\right\rfloor\right]}, A_{2}=N_{\left(k-2,\left\lfloor\frac{j}{2^{2}}\right\rfloor\right)}, A_{i}=N_{\left(k-i,\left\lfloor\frac{j}{2^{i}}\right\rfloor\right)}, \cdots, A_{k}=N_{\left(0,\left\lfloor\frac{j}{2^{k}}\right\rfloor\right)}=N_{(0,0)}
$$

Then

$$
\begin{aligned}
& N_{(1,1-j \bmod 2)}^{A_{1}}=N_{\left(k, 2\left\lfloor\frac{j}{2}\right\rfloor+1-j \bmod 2\right)}, N_{\left(2,2^{2}-1-j \bmod 2^{2}\right)}^{A_{2}}=N_{\left(k, 2^{2}\left\lfloor\frac{j}{2^{2}}\right\rfloor+2^{2}-1-j \bmod 2^{2}\right)}, \cdots \\
& N_{\left(i, 2^{i}-1-j \bmod 2^{i}\right)}^{A_{i}}=N_{\left(k, 2^{i}\left\lfloor\frac{j}{2^{i}}\right\rfloor+2^{i}-1-j \bmod 2^{i}\right)}, \cdots, N_{\left(k, 2^{k}-1-j \bmod 2^{k}\right)}^{A_{k}}=N_{\left(k, 2^{k}\left\lfloor\frac{j}{2^{k}}\right\rfloor+2^{k}-1-j \bmod 2^{k}\right)}
\end{aligned}
$$

By formula $a \bmod b=a-b\left\lfloor\frac{a}{b}\right\rfloor$, it holds

$$
\begin{aligned}
& N_{\left(1,2\left\lfloor\frac{j}{2}\right\rfloor+1-j\right)}^{A_{1}}=N_{\left.\left(k, 2^{1+1}\left\lfloor\frac{j}{2}\right\rfloor+1-j\right)\right)}, N_{\left(2,2^{2}\left\lfloor\frac{j}{2^{2}}\right\rfloor+2^{2}-1-j\right)}^{A_{2}}=N_{\left(k, 2^{2+1}\left\lfloor\frac{j}{2^{2}}\right\rfloor+2^{2}-1-j\right)}, \ldots, \\
& N_{\left(i, 2^{i}\left\lfloor\frac{j}{2^{i}}\right\rfloor+2^{i}-1-j\right)}^{A_{i}}=N_{\left(k, 2^{i+1}\left\lfloor\frac{j}{2^{i}}\right\rfloor+2^{i}-1-j\right)}, \ldots, N_{\left(k, 2^{k}\left\lfloor\frac{j}{2^{k}}\right\rfloor+2^{k}-1-j\right)}^{A_{k}}=N_{\left(k, 2^{k+1}\left\lfloor\frac{j}{2^{k}}\right\rfloor+2^{k}-1-j\right)}
\end{aligned}
$$

Example 2 Take node $N_{(5,13)}$ that leads to $k=5$ and $j=13$. The ancestors of $N_{(5,13)}$ are $A_{1}=N_{(4,6)}, A_{2}=N_{(3,3)}, A_{3}=N_{(2,1)}$, $A_{4}=N_{(1,0)}$ and $A_{5}=N_{(0,0)}$. In $T_{A_{1}}, N_{(5,13)}=N_{(1,13 \bmod 2)}^{A_{1}}=N_{(1,1)}^{A_{1}}$ and its symmetric brother is $N_{\left(1,2^{1}-1-13 \bmod 2\right)}^{A_{1}}=N_{(1,0)}^{A_{1}}=$ $N_{\left(5,2\left\lfloor\frac{13}{2}\right\rfloor+2^{1}-1-13 \bmod 2\right)}=N_{(5,12)}$; in $T_{A_{2}}, N_{(5,13)}=N_{\left(2,13 \bmod 2^{2}\right)}^{A_{2}}=N_{(2,1)}^{A_{2}}$ and its symmetric brother is $N_{\left(2,2^{2}-1-13 \bmod 2^{2}\right)}^{A_{2}}=$ $N_{(2,2)}^{A_{2}}=N_{\left(5,2^{2} \times\left\lfloor\frac{13}{2^{2}}\right\rfloor+2^{2}-1-13 \bmod 2^{2}\right)}=N_{(5,14)} ;$ in $T_{A_{3}}, N_{(5,13)}=N_{\left(3,13 \bmod 2^{3}\right)}^{A_{3}}=N_{(3,5)}^{A_{3}}$ and its symmetric brother is $N_{\left(3,2^{3}-1-13 \bmod 2^{3}\right)}^{A_{3}}$ $=N_{(3,2)}^{A_{3}}=N_{\left(5,2^{3} \times\left\lfloor\frac{13}{2^{3}}\right]+2^{3}-1-13 \bmod 2^{3}\right)}=N_{(5,10)}$; in $T_{A_{4}}, N_{(5,13)}=N_{\left(4,13 \bmod 2^{4}\right)}^{A_{4}}=N_{(4,13)}^{A_{4}}$ and its symmetric brother is $N_{\left(4,2^{4}-1-13 \bmod 2^{4}\right)}^{A_{4}}=N_{(4,4)}^{A_{4}}=N_{\left(5,2^{4} \times\left\lfloor\frac{13}{2^{4}}\right\rfloor+2^{4}-1-13 \bmod 2^{4}\right)}=N_{(5,2)}$; in $T_{A_{5}}, N_{(5,13)}=N_{\left(5,13 \bmod 2^{5}\right)}^{A_{5}}=N_{(5,13)}^{A_{5}}$ and its symmetric brother is $N_{\left(5,2^{5}-1-13 \bmod 2^{5}\right)}^{A_{5}}=N_{(5,18)}^{A_{5}}=N_{\left(5,2^{5} \times\left\lfloor\frac{13}{2^{5}}\right\rfloor+2^{5}-1-13 \bmod 2^{5}\right)}=N_{(5,18)}$. 


\section{Conclusion}

Calculation of the ancestors, the descendants and the brothers of a node in binary tree is by nature a conventional task in discrete mathematics and computer science. Since the computation also concerns many engineering applications, the related study has been concerned. The theorems proved in this article can undoubtedly provide new criterions for certain computations. Hope it is helpful.

\section{Acknowledgment}

The research work is supported by the State Key Laboratory of Mathematical Engineering and Advanced Computing under Open Project Program No.2017A01, Department of Guangdong Science and Technology under project 2015A010104011, Foshan Bureau of Science and Technology under projects 2016AG100311. The authors sincerely present thanks to them all.

\section{References}

Paul, E. B. (2004). Dictionary of Algorithms and Data Structures. US National Institute of Standards and Technology.

Rosen, K. H. (2000). Handbook of discrete and combinatorial mathematics. CRC Press.

Dinesh, P. M., \& Sartaj, S. (2004). Handbook of Data Structures and Applications. Crc Press.

Berkman, O., \& Vishkin, U. (1994). Finding level-ancestors in trees. Journal of Computer E System Sciences, 48(2), 214-230.

Papamichail, D., Caputi, T.,\& Papamichail, G. (2014). The Level Ancestor Problem in Practice. arXiv:1402.2741

Knight, S., Kim, H., Meghanathan, N, \& et al. (2007). Fast Determination of Ancestor-Descendant Relationships using Bit Patterns. International Conference on Information $\mathcal{E}$ Knowledge Engineering, Ike 2007, 178-182.

Fly, P., Meghanathan, N., \& Isokpehi, R. (2011). Implementation of the Binary Coding Scheme and the Tree Traversal Algorithms to Test for Ancestor-descendant Relationship in K-ary Trees. International Journal of Research $\mathcal{E}$ Reviews in Applied Sciences, 8(3), 386-394.

WANG, X. B. (2015). Analytic Formulas for Computing LCA and Path in Complete Binary Trees. International Journal of Scientific and Innovative Mathematical Research, 3(4), 1-8.

\section{Copyrights}

Copyright for this article is retained by the author(s), with first publication rights granted to the journal.

This is an open-access article distributed under the terms and conditions of the Creative Commons Attribution license (http://creativecommons.org/licenses/by/4.0/). 